Research Paper

\title{
Candidate chemosensory Genes in Female Antennae of the Noctuid Moth Spodoptera littoralis
}

\author{
Emmanuelle Jacquin-Joly $1,2, \llbracket$, Fabrice Legeai $^{3}$, Nicolas Montagnée ${ }^{1,2}$, Christelle Monsempes ${ }^{1,2}$, Ma- \\ rie-Christine François ${ }^{1,2}$, Julie Poulain ${ }^{4}$, Frédéric Gavory ${ }^{4}$, William B. Walker III5, Bill S. Hansson ${ }^{6}$ and Mat- \\ tias C. Larsson ${ }^{5}$
}

1. INRA, UMR-A 1272 Physiologie de l'Insecte : Signalisation et Communication, route de Saint-Cyr, F-78026 Versailles Cedex, France.

2. UPMC-Université Paris 6, UMR-A 1272, Physiologie de l'Insecte : Signalisation et Communication, 7 quai Saint-Bernard, F-75252 Paris Cedex 05, France.

3. IRISA, équipe Symbiose, Campus universitaire de Beaulieu, F-35042 Rennes Cedex, France.

4. Genoscope, Centre National de Séquençage, 2 rue Gaston Crémieux, BP 191, F-91057 Evry Cedex, France.

5. Department of Plant Protection Biology, Division of Chemical Ecology, Swedish University of Agricultural Sciences, Alnarp, Sweden.

6. Department of Evolutionary Neuroethology, Max Planck Institute for Chemical Ecology, Hans Knoell Strasse 8, D-07745 Jena, Germany.

$\bowtie$ Corresponding author: Emmanuelle Jacquin-Joly, Phone: +33 1308332 12, Fax: +33 13083 31 19, Email: emmanuelle.jacquin@versailles. inra.fr.

( ) Ivyspring International Publisher. This is an open-access article distributed under the terms of the Creative Commons License (http://creativecommons.org/ licenses/by-nc-nd/3.0/). Reproduction is permitted for personal, noncommercial use, provided that the article is in whole, unmodified, and properly cited.

Received: 2012.04.14; Accepted: 2012.07.31; Published: 2012.08.10

\begin{abstract}
Chemical senses are crucial for all organisms to detect various environmental information. Different protein families, expressed in chemosensory organs, are involved in the detection of this information, such as odorant-binding proteins, olfactory and gustatory receptors, and ionotropic receptors. We recently reported an Expressed Sequence Tag (EST) approach on male antennae of the noctuid moth, Spodoptera littoralis, with which we could identify a large array of chemosensory genes in a species for which no genomic data are available.

Here we describe a complementary EST project on female antennae in the same species. 18,342 ESTs were sequenced and their assembly with our previous male ESTs led to a total of 13,685 unigenes, greatly improving our description of the S. littoralis antennal transcriptome. Gene ontology comparison between male and female data suggested a similar complexity of antennae of both sexes. Focusing on chemosensation, we identified 26 odorant-binding proteins, 36 olfactory and 5 gustatory receptors, expressed in the antennae of $S$. littoralis. One of the newly identified gustatory receptors appeared as female-enriched. Together with its atypical tissue-distribution, this suggests a role in oviposition. The compilation of male and female antennal ESTs represents a valuable resource for exploring the mechanisms of olfaction in S. littoralis.
\end{abstract}

Key words: Olfactory receptor, Gustatory receptor, Odorant-binding protein, Expressed sequence tag, Lepidoptera, Spodoptera littoralis.

\section{Introduction}

The sense of smell is highly important for most animals to detect chemical information regarding various fitness-related resources. For many decades, moths have proven to be important models for studying the physiological and molecular bases of olfactory detection [1]. First, their nocturnal life has led to a 
highly developed and sensitive olfactory system well suited to describe general paradigms. Second, moths include diverse and important pests of crops, forests and stored products, and studying their sense of smell offers the possibility to develop olfactory-based strategies to perturb critical behaviors, such as sex pheromone-mediated reproduction, host selection and oviposition.

The noctuid moth Spodoptera littoralis is one of the major Lepidoptera models used in olfaction research. This polyphagous noctuid species is an important cotton pest, and extensive research has led to a comprehensive insight into its chemical communication system. The sex pheromone and plant volatiles activating olfactory sensory neurons (OSN) have been identified [2, 3], various functional types of antennal olfactory sensilla have been characterized [3, 4], and the organization of the primary central olfactory system, the antennal lobe, as well as higher areas as the mushroom bodies has been described [5-7]. From a molecular point of view, we recently sequenced an Expressed sequence tag (EST) library prepared from male $S$. littoralis antennae, leading to the identification of a partial repertoire of genes putatively involved in odorant and pheromone detection [8]. Among them, we described members of crucial gene families involved in the olfactory process, such as odorant-binding proteins (OBPs), olfactory and gustatory receptors (ORs, GRs), and ionotropic receptors (IRs). OBPs are soluble proteins proposed to bind odorant molecules and transport them through the aquaeous sensillar lymph, allowing hydrophobic molecules to reach OSN dendrites $[9,10]$. Another family of binding proteins, the chemosensory protein (CSP) family, groups soluble proteins whose function is unclear. Although some exhibit binding activity towards odorants and pheromones $[11,12]$, they may participate in other physiological processes beyond chemoreception $[13,14]$. ORs and IRs constitute two families of chemosensory receptors located in the dendritic membrane of OSNs, whose activation upon ligand binding leads to the generation of an electrical signal that is transmitted to the brain $[15,16]$. ORs are multi-transmembrane domain ionotropic receptors, functioning as ion channels via heterodimerization with a subunit conserved within insects, referred to as ORco [17-19], while the variable part of the dimer defines ligand specificity $[20,21]$. This variable part also couples to $G$ proteins, inducing upon activation a complementary metabotropic signaling [20]. IRs are related to ionotropic glutamate receptors but with a divergent ligand-binding domain [16]. Although they appear to be far more ancient than ORs, as they are found across protostomians, they were only recently discovered as key actors in odorant detection $[22,23]$. GRs are transmembrane domain receptors distantly related to insect ORs and expressed in gustatory receptor neurons. Recent studies suggest that GRs present a similar membrane topology to ORs [24] and act as ionotropic channels [25].

Research in the molecular field of moth olfaction has, until recently, largely been restricted to the silkmoth Bombyx mori, due to the availability of genomic data. Our EST strategy has been demonstrated to be well suited to identify a wide array of olfactory genes in a given species and was particularly relevant for the identification of divergent chemosensory receptors in a species for which no genomic data is available [8]. Similar strategies have been followed to identify broad or partial repertoires of chemosensory receptors in some other moth species, such as Epiphyas postvittana [26], Manduca sexta [27] and Cydia pomonella [28], but the picture of the moth OR and IR gene families is still incomplete.

Here, we report the sequencing and analyses of a female $S$. littoralis antennal EST library that, together with our previous male EST data, allows us to extend our view of the $S$. littoralis antennal transcriptome and to significantly increase the number of identified olfactory genes in S. littoralis. Both male and female ESTs were assembled, leading to a total of 13,685 unigenes whose gene ontology (GO) annotation revealed enrichment in binding and catalytic activities. These data allowed us to identify new S. littoralis olfactory genes, including binding proteins and chemosensory receptors. Further qPCR experiments were conducted to study tissue- and sex-distribution of the newly identified ORs and GRs, revealing one female-enriched transcript possibly involved in oviposition.

\section{Materials and Methods}

\section{Insect rearing and female antennae cDNA li- brary construction}

Insects were from a laboratory strain of S. littoralis that originated from an Egyptian population. Insects were reared on semi-artificial diet [29], under $23^{\circ} \mathrm{C}, 60-70 \%$ relative humidity and $16: 8$ light:dark cycle. Pupae were sexed and males and females were kept separately. Antennae were collected from 1-2 day old naïve adult females and stored at $-80^{\circ} \mathrm{C}$. Total RNA were isolated using the TriZol reagent (Invitrogen, Carlsbad, CA, USA), quantified in a spectrophotometer, and the quality verified by agarose gel electrophoresis. A custom normalized cDNA library was created by Evrogen (Russia) in the pAL 17.3 vector, using $1 \mathrm{mg}$ of total RNA as starting material, without 
any amplification. The normalization procedure was used to minimize EST redundancy and to enrich the library for rare and low abundant genes, to allow new gene discovery.

\section{EST sequencing}

The library was plated, and 20,000 clones were randomly picked. Their 5 ' ends were sequenced using REV primer by the Genoscope (Evry, France). The plated library was arrayed robotically and bacterial clones had their plasmid DNA amplified using phi29 polymerase. The plasmids were end-sequenced using BigDye Termination kits on Applied Biosystems 3730xl DNA Analysers. Adaptor and vector were localized using cross_match (http://www.phrap.org/) using default matrix ( 1 for a match, -2 penalty for a mismatch), with mean scores of 6 and 10, respectively. Sequences were then trimmed following three criteria: vector and adaptor, poly(A) tail or low quality (defined as at least 15 among $20 \mathrm{bp}$ with a phred score below 12). We finally obtained the $5^{\prime}$ end sequences of 18,342 ESTs.

\section{Sequence processing, assembly, unigene and peptide generation}

Female ESTs were assembled with previously obtained male ESTs using the TGI Clustering tools (TGICL, http://compbio.dfci.harvard.edu/tgi/ software) using the default parameters (minimum percent identity for overlaps: 94, minimum overlap length: $30 \mathrm{bp}$, maximum length of unmatched overhangs: 30), generating unigenes and singletons. Peptides were extracted from the unigenes using FrameDP 1.03 [30], with three training iterations and using Swissprot (398,181 entries, August 2009) as reference protein database.

\section{Gene identification and functional annotation}

The newly identified unigenes were compared to the NCBI non redundant protein database (June $24^{\text {th }}$ 2011 version), using BLASTX, with a 1e-8 e-value threshold. The Gene Ontology mapping and distribution were done with the help of BLAST2GO (GO association done by a BLAST against the NCBI NR database) [31]. Finally, the functional domain protein profile and domain were predicted by queries against InterPro using InterproScan, [32], running a batch of analyses (BLASTProDom, Coil, FprintScan, Gene3D, HMMPanther, HMMPfam, HMMPIR, HMMSmart, HMMTigr, PatternScan, ProfileScan, RNA-BINDING, Seg and Superfamily) on the predicted ORFs.

\section{GO-term enrichment}

The analysis of the enrichment in GO-term be- tween male and female transcriptomes was performed with the BLAST2GO application using GOSSIP [33]. GO-terms in female data (female singletons + contigs assembled from female ESTs only) (test group) were tested for enrichment compared to male data (male singletons + contigs assembled from male ESTs only) (reference group) using Fisher's exact test with multiple testing correction.

\section{Identification of odorant-binding proteins and chemosensory receptors}

The $S$. littoralis antennal unigenes were searched with B. mori OBPs, CSPs, chemosensory receptors and available insect ORs retrieved from Swissprot as queries using TBLASTN [34]. Additionally, the Interproscan results were scanned for the Interpro accession IPR006170 (Pheromone/general odorant-binding protein, PBP/GOBP Molecular Function: odorant binding GO:0005549) and IPR004117 (Molecular Function: olfactory receptor activity GO:0004984). S. littoralis putative chemosensory receptor sequences were in turn employed in searches to find more genes in an iterative process. OR transmembrane domains were predicted using the TMHMM server v.2.0 [35]. OBPs and CSPs were searched for the occurrence of a signal peptide using SignalP 4.0 [36], secondary structures were predicted using the Psipred server [37], and logos were generated using WebLogo [38].

\section{Phylogenetic analyses}

We built OR and GR neighbor-joining trees based on Lepidoptera data sets. The OR data set contained 64 amino acid sequences from B. mori [39], 18 sequences from $H$. virescens [40, 41], 45 sequences from $M$. sexta [27] and the three OR sequences characterized in Epiphyas postvittana [26]. The GR data set contained 65 amino acid sequences from B. mori [42], three, two and one sequences from $H$. virescens [41], M. sexta [27] and Papilio xuthus [43], respectively. Amino acid sequences were aligned using ClustalW2 [44]. Unrooted trees were constructed using the BioNJ algorithm with Poisson correction of distances, as implemented in Seaview v.4 [45]. Node support was assessed using a bootstrap procedure based on 1000 replicates. Images were created using the iTOL web server [46].

\section{Quantitative real-time PCR}

Naïve males and females in the middle of their second scotophase were used in the following experiments: female antennae, brains, proboscis, legs (mixture of front, middle, and hind legs), thorax, ovipositors and male antennae total RNAs (one sample per tissue) were extracted with the RNeasy ${ }^{\circledR} \mathrm{Mi}$ - 
croKit (Qiagen, Hilden, Germany) that included a column DNase treatment. For each tissue, single-stranded cDNA was synthesized from $1 \mu \mathrm{g}$ of total RNA with $200 \mathrm{U}$ of M-MLV reverse transcriptase (Clontech, Mountain View, CA, USA) using buffer and protocol supplied in the Advantage ${ }^{\circledR}$ RT-for-PCR kit (Clontech). Gene-specific primers for S. littoralis chemosensory receptors and the endogenous control $r p L 8$ were designed using the Beacon Designer 4.0 software (Bio-Rad, Hercules, CA, USA), yielding PCR products ranging from 100 to $250 \mathrm{bp}$ (Table 1). qPCR mix was prepared in a total volume of $20 \mu \mathrm{l}$ with $10 \mu \mathrm{l}$ of Absolute QPCR SYBR Green Mix (ThermoFisher Scientific, Epsom, UK), $5 \mu$ l of diluted cDNA (or water for the negative control or RNA for controlling for the absence of genomic DNA) and $200 \mathrm{nM}$ of each primer.
qPCR assays were performed on S. littoralis cDNAs using a MJ Opticon Monitor Detection System (Bio-Rad). The PCR program began with a cycle at 95 ${ }^{\circ} \mathrm{C}$ for $15 \mathrm{~min}$, followed by 40 cycles of $20 \mathrm{~s}$ at $95^{\circ} \mathrm{C}, 15$ s at 53 to $62{ }^{\circ} \mathrm{C}$ (depending on the primer pair) and 20 $\mathrm{s}$ at $72{ }^{\circ} \mathrm{C}$. To assess the purity of the PCR reactions, a dissociation curve of the amplified product was performed by gradual heating from $50^{\circ} \mathrm{C}$ to $95^{\circ} \mathrm{C}$ at 0.2 ${ }^{\circ} \mathrm{C} / \mathrm{s}$. Standard curves were generated by a five-fold dilution series of a cDNA pool evaluating primer efficiency $E\left(E=10^{(-1 / \text { slope })}\right)$. All reactions were performed in duplicate. Chemosensory receptor expression levels were calculated relative to the expression of the $r p L 8$ control gene and expressed as the ratio = $\mathrm{E}_{\text {SlitCR }}\left(\Delta \mathrm{CT}\right.$ SlitCR) $/ \mathrm{E}_{\mathrm{rpL} 8}(\Delta \mathrm{CT} \mathrm{rpL}$ 8) [47].

Table I. Forward and reverse primer sequences used in real-time PCR, annealing temperatures, resulting amplicon lengths and PCR efficiencies.

\begin{tabular}{|c|c|c|c|c|c|}
\hline Unigenes & $\begin{array}{l}\text { qPCR Forward primer sequences } \\
\left.\text { (5' to } 3^{\prime}\right)\end{array}$ & $\begin{array}{l}\text { qPCR Reverse primer sequences } \\
\left.\text { (5' to } 3^{\prime}\right)\end{array}$ & Annealing $\mathrm{T}\left({ }^{\circ} \mathrm{C}\right)$ & $\begin{array}{l}\text { Amplicon lenght } \\
(\mathrm{pb})\end{array}$ & Efficiency \\
\hline SlitOR7 & ССTTCCTATCGATGGCTCTG & CCCAGGTACCACTTGCAGTT & 60 & 115 & 2.1 \\
\hline SlitOR10 & TTGCACTTTATGGGCAATGA & GAAGAGGAAAAGCGCTGATG & 62 & 188 & 1.9 \\
\hline SlitOR12 & TTGGCCTTGGGTGTATCTTC & AAACGGCCACAAGTCTCATC & 62 & 171 & 2.1 \\
\hline SlitOR19 & AAACGTGACTCCGTGAGCTT & CCGCCATCAACGTATTTTCT & 62 & 148 & 2.3 \\
\hline SlitOR24 & CGCATCCGTTTATCGACTTT & CAAACCAGACCACAAGAGCA & 60 & 116 & 2.1 \\
\hline SlitOR32 & GGTACTAAGGCGGTGGATGA & ССААТССАСААССААААТСС & 58 & 192 & 2.2 \\
\hline SlitOR34 & CGCAATATGGGTGTCTTCCT & CATGTTGCTCGATTCCCTTT & 62 & 178 & 2.3 \\
\hline SlitGR1 & CGACATTTACCGCGAATTTT & TTGGGACGAGCCTCAATTAC & 60 & 114 & 2.1 \\
\hline SlitGR2 & GCCGGTGTCCAAGATACACT & CATGCTGATTGCCGAAGTAA & 62 & 168 & 2.2 \\
\hline SlitGR4 & ATGCTGCGTCACACGACTAC & CCAACGGGAACATCTTCAAT & 58 & 115 & 2.2 \\
\hline SlitGR5 & GTTTGTGTTGCTGGTGATGG & TTCGAGGCTAGGATCAAGGA & 62 & 159 & 1.9 \\
\hline S. littoralis RpL8 & ATGCCTGTGGGTGCTATGC & TGCCTCTGTTGCTTGATGGTA & $58 / 62$ & 189 & 1.90 \\
\hline
\end{tabular}

\section{Results and discussion}

Complete repertoires of moth olfactory genes have been established only in B. mori, thanks to the availability of its sequenced genome. To date, it remains the only moth genome available. RNA sequencing appears to be a good alternative to identify partial olfactory gene repertoires in other species, and previous studies have proved it to be efficient $[8,27]$. In particular, the high sequence divergence observed within insect ORs has long served as a brake in chemosensory receptor identification. These receptors are very divergent both within and across insect species, precluding their identification through classical homology-based approaches [48].

\section{EST statistics and unigene prediction}

A total of 18,342 ESTs (mean length: $664.9 \mathrm{bp}$, median length: $665.1 \mathrm{bp}$, max length: $888 \mathrm{bp}$, min length: $72 \mathrm{bp}$, Table 2) were obtained from female antennae of $S$. littoralis. These ESTs have been deposited in the European Molecular Biology Laboratory database (EMBL) [EMBL:FQ958213-FQ976554]. The ESTs were processed and assembled with 20,760 male ESTs previously obtained [8]. 30,986 ESTs were assembled into 5,623 contigs, 8,062 ESTs were singletons (20.6\% of total ESTs). On average, each contig was assembled from 18 ESTs. Among the singletons, 4,361 corresponded to male and 3,701 to female ESTs. The assembly led to a total of 13,685 unigenes (mean length: $980.9 \mathrm{bp}$, median length: $832 \mathrm{bp}$, max length: 4,100 bp, min length: $40 \mathrm{bp}$, Table 2) that putatively 
represent different transcripts. Compared to the 9,033 unigenes we previously identified in male antennae [8], this led to the identification of 4,652 new unigenes from S. littoralis antennae. It must be pointed out that we did only $5^{\prime}$ end sequencing that, together with splice variants, polymorphism or reverse transcriptase errors, may have led to under-assembly and thus over-estimation of unigene counts. Indeed, by using the mean coding sequence size of 1.1-1.2 kb in the $B$. mori genome as reference [49], we could estimate the number of genes expressed to be 11,000-12,000 (13,685 unigenes with an average size of $\sim 981 \mathrm{pb}$ ). This is higher than the estimation made in $M$. sexta, for which $7,000-8,000$ genes were estimated to be expressed [27].

\section{Identification of putative ORFs}

Among the 13,685 unigenes, 8,449 presented a coding region (62\%, mean length: 205.6 aa, median length: 203 aa, max length: 922 aa, min length: 30 aa, Table 2). Protein sequences translated from the predicted open reading frame (ORF) set were compared to the non-redundant protein database (NR, version October $\left.26^{\text {th }} 2011\right)$. Most of the sequences (80.3\%) translated from predicted ORFs showed similarity to known proteins. 1,663 ORFs showed no similarity at all.

\section{Gene identification and functional annotation}

Fig. 1 illustrates the distribution of the S. littoralis unigene set in GO terms. Among the 13,685 S. littoralis unigenes, 3,376 correspond to at least one GO-term. As observed in the antennal transcriptome of $M$. sexta [27], a large number of transcripts could not be associated with a GO-term (75 \%). Among those associated to a GO-term, 2,944 were assigned to a molecular function $(87.2 \%), 2,147$ to putative biological processes $(63.6 \%)$, and 1,673 to a cellular component (49.6 $\%)$ (Fig. 1). In the molecular function category, the terms "binding" and "catalytic activity" were the most represented (54\% and $47 \%$, respectively), as already observed through the analyses of the male ESTs [8]. When we compared the GO-term distribution of "male only" sequences (male singletons + contigs assembled from male ESTs only) with "female only", differential distribution $(P<0.05)$ was observed for some GO-terms, such as "signal transduction" enriched in male antennae (Fig. 2). Interestingly, numbers of GO-terms were similar in the two datasets, suggesting a similar complexity of male and female antennae (Supplementary material S1). This is different from what has been observed in the analysis of $M$. sexta male and female transcriptomes [27], where the number of terms appearing for biological process and molecular function in female antennae was approximately twice the number as for male antennae.
Molecular function

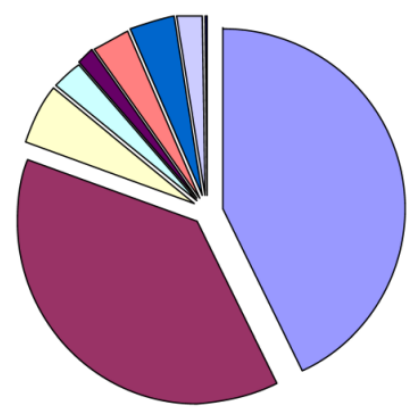

$\square$ binding (1835)

$\square$ catalytic activity (1612)

$\square$ transporter activity (227)

$\square$ transcription regulator activity (113)

$\square$ enzyme regulator activity (73)

$\square$ structural molecule activity (147)

$\square$ molecular transducer activity (178)

$\square$ electron carrier activity (78)

antioxidant activity (13)

$\square$ protein tag (1)
Biological process

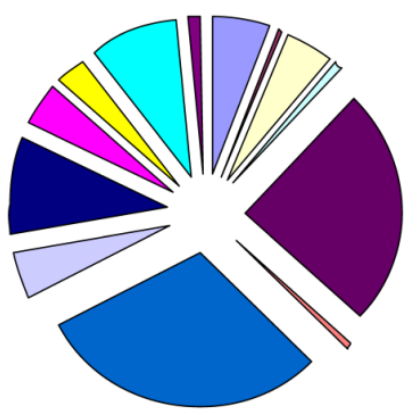

$\square$ cellular component organization (291)

$\square$ growth (31)

$\square$ signaling (234)

$\square$ death (39)

cellular process (1189)

$\square$ cell proliferation (24)

$\square$ metabolic process (1465)

$\square$ developmental process (233)

口 biological regulation (473)

$\square$ multicellular organismal process (214)

$\square$ response to stimulus (152)

$\square$ localization (432)

$\square$ reproduction $(\mathbf{8 2})$

\section{Cellular component}

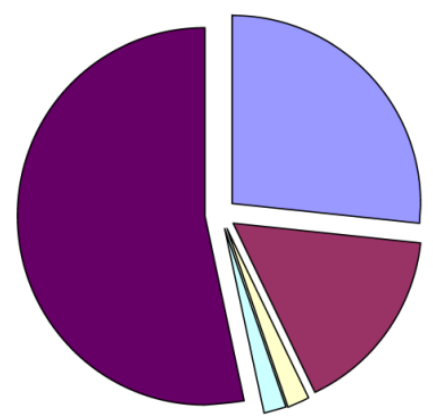

$\square$ organelle (806)

$\square$ macromolecular complex (488)

$\square$ extracellular region (62)

$\square$ membrane enclosed lumen (56)

$\square$ cell (1614)

Figure I. Distribution of S. littoralis unigenes annotated at GO level 2. 
Table 2. Data summary.

\begin{tabular}{|c|c|c|c|c|c|c|}
\hline & Counts (total nb) & $\begin{array}{l}\text { Min. length } \\
\text { (bp/aa) }\end{array}$ & $\begin{array}{l}\text { Average length } \\
\text { (bp/aa) }\end{array}$ & Max length (bp/aa) & Median lenght (bp/aa) & Accession numbers \\
\hline \multirow[t]{3}{*}{ Male ESTs } & 20760 & 40 & 958.1 & 1525 & 820 & FQ0142366-FQ032656 \\
\hline & & & & & & GW824594-GW826804 \\
\hline & & & & & & HO118288-HO118415 \\
\hline Female ESTs & 18342 & 72 & 664.9 & 888 & 665.1 & FQ958213-FQ976554 \\
\hline Unigenes & 13685 & 40 & 980.9 & 4,100 & 832 & \\
\hline Coding regions & 8449 & 30 & 205.6 & 922 & 203 & \\
\hline
\end{tabular}

\section{Differential GO-term distribution (\% of sequences)}

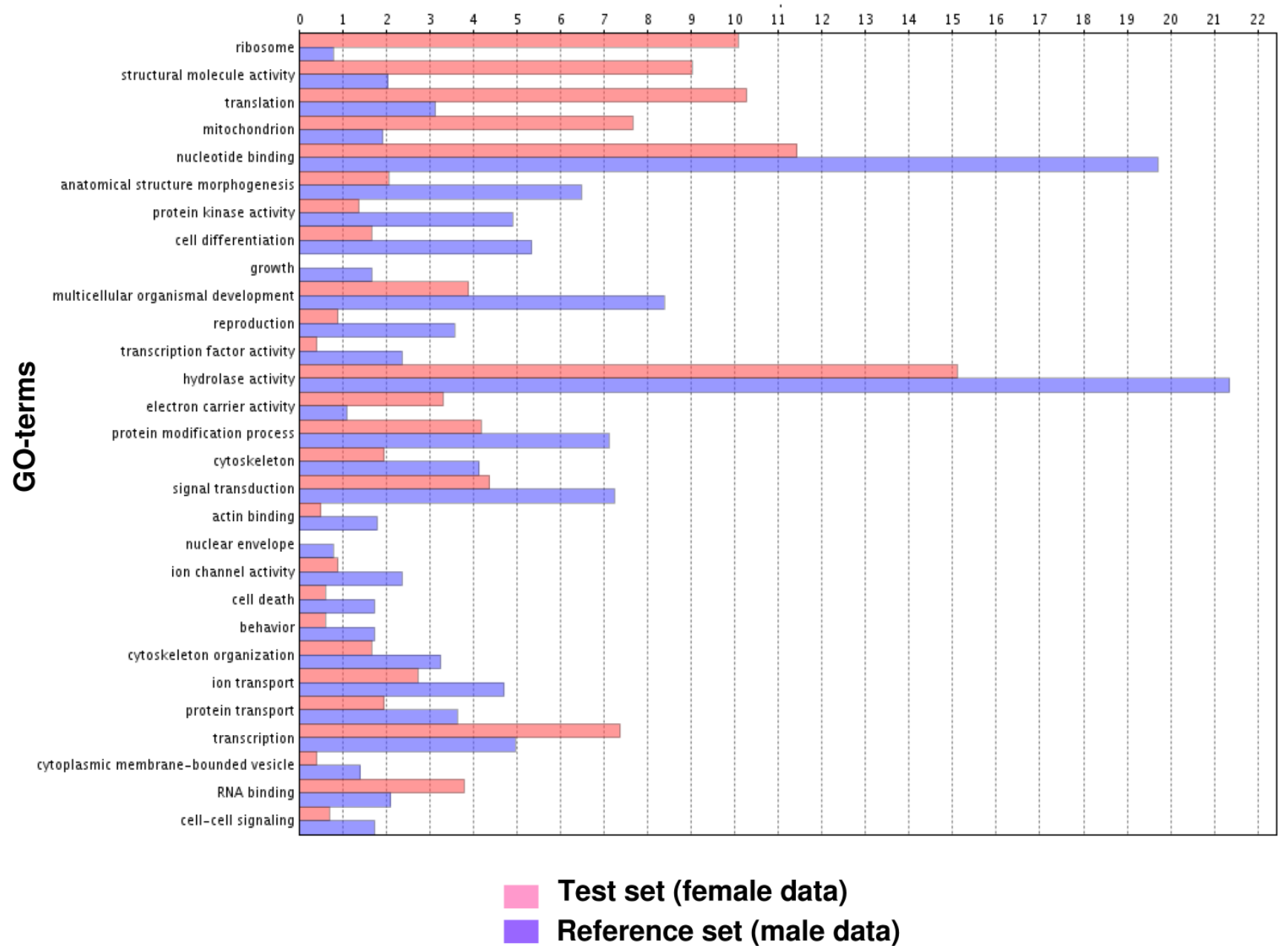

Figure 2. GO-terms (GO-Slim) differentially distributed between male and female transcriptomes (Fisher's exact test, $\mathrm{P}<0.05)$. Female data: female singletons + contigs assembled from female ESTs only (test group). Male data: male singletons + contigs assembled from male ESTs only (reference group).

\section{Olfactory and gustatory receptors}

In our previous study, which focused on male antennal ESTs, we annotated 29 ORs and 2 GRs. Thanks to the female antennal sequences, we could extend five of the previously characterized SlitORs (OR4, 5, 8, 21, 29, former accession numbers FQ018861, EZ982621, FQ030158, EZ983645, EZ981024) and we identified seven new candidate SlitORs (OR7, $10,12,19,24,32,34)$. In addition, one GR (GR2, former 
accession number GW825869) could be extended and three new candidate GRs were annotated (GR1, 4, 5). This led to a total of 36 ORs and 5 GRs identified in $S$. littoralis antennae (Table 3, fasta format file in Supplementary material S2). For convenience, SlitORs and GRs were numbered according to their closest homologs -when possible - from $H$. virescens, $M$. sexta or B. mori in the phylogenetic analyses (Fig. $3 \& 4$ ). Note that SlitOR9 is missing because the corresponding sequence was first annotated as a candidate OR but was later eliminated due to the presence of a stop codon within the predicted ORF.

Table 3. List of S. littoralis unigenes putatively involved in chemosensory reception. Transmembrane domains (TM) were predicted using TMHMM version v.2.0.[35].

\begin{tabular}{|c|c|c|c|c|}
\hline Name & Length (amino acid) & $\mathrm{TM}$ nb & BlastP hit & E value \\
\hline SlitOR1 & 298 & 4 & ref |NP_001116817.1 | olfactory receptor-like [Bombyx mori] & 1e-118 \\
\hline SlitOR2 & 473 & 7 & gb |ABQ82137.1 | chemosensory receptor 2 [Spodoptera littoralis] & 0.0 \\
\hline SlitOR3 & 270 & 4 & gb | AEF32141.1| odorant receptor [Spodoptera exigua] & $8 \mathrm{e}-174$ \\
\hline SlitOR4 & 386 & $6-7$ & ref |NP_001166616.1 | olfactory receptor 54 [Bombyx mori] & 2e-101 \\
\hline SlitOR5 & 397 & 6 & ref | NP_001103623.1 | olfactory receptor 33 [Bombyx mori] & $8 e-99$ \\
\hline SlitOR6 & 263 & 3 & emb |CAG38117.1 | putative chemosensory receptor 16 [Heliothis virescens] & $5 e-111$ \\
\hline SlitOR7 & 216 & 4 & emb |CAD31853.1 | putative chemosensory receptor 7 [Heliothis virescens].. & $6 e-98$ \\
\hline SlitOR8 & 258 & 4 & emb |CAD31949.1 | putative chemosensory receptor 8 [Heliothis virescens] & 2e-110 \\
\hline SlitOR10 & 212 & 3 & gb | ACC63238.1| olfactory receptor 10 [Helicoverpa armigera] & 1e-70 \\
\hline SlitOR11 & 223 & 3 & gb | ACS45305.1 | candidate odorant receptor 2 [Helicoverpa armigera] & 1e-139 \\
\hline SlitOR12 & 224 & 3 & emb|CAG38113.1 | putative chemosensory receptor 12 [Heliothis virescens]. & $8 \mathrm{e}-79$ \\
\hline SlitOR13 & 299 & 4 & dbj| BAG71423.2 | olfactory receptor [Mythimna separata] & $5 e-130$ \\
\hline SlitOR14 & 238 & 4 & ref |NP_001155301.1 | olfactory receptor 60 [Bombyx mori] & $8 \mathrm{e}-126$ \\
\hline SlitOR15 & 390 & 7 & ref | NP_001091789.1 | olfactory receptor 15 [Bombyx mori] & 1e-156 \\
\hline SlitOR16 & 410 & $7-8$ & emb |CAG38117.1 | putative chemosensory receptor 16 [Heliothis .virescens] & 0.0 \\
\hline SlitOR17 & 391 & 5 & emb |CAG38118.1 | putative chemosensory receptor 17 [Heliothis virescens]. & 0.0 \\
\hline SlitOR18 & 328 & 5 & gb|ACL81189.1 | putative olfactory receptor 18 [Spodoptera littoralis] & 0.0 \\
\hline SlitOR19 & 216 & 4 & tpg|DAA05980.1|TPA: TPA_exp: odorant receptor 22 [Bombyx mori] & 1e-101 \\
\hline SlitOR20 & 331 & 5 & emb|CAD31949.1 | putative chemosensory receptor 8 [Heliothis virescens] & $4 e-108$ \\
\hline SlitOR21 & 215 & 4 & emb |CAG38122.1 | putative chemosensory receptor 21 [Heliothis virescens] & $5 e-112$ \\
\hline SlitOR22 & 280 & 5 & dbj|BAH66361.1 | olfactory receptor [Bombyx mori] & $5 e-07$ \\
\hline SlitOR23 & 422 & 6 & gb | EHJ75140.1 | olfactory receptor [Danaus plexippus] & $4 e-63$ \\
\hline SlitOR24 & 211 & 5 & ref |NP_001166621.1 | olfactory receptor 64 [Bombyx mori] & $4 e-42$ \\
\hline SlitOR25 & 131 & 1 & ref | NP_001166621.1 | olfactory receptor 64 [Bombyx mori] & $8 e-58$ \\
\hline SlitOR26 & 391 & 5 & ref |NP_001091790.1 | candidate olfactory receptor [Bombyx mori] & 0.0 \\
\hline SlitOR27 & 429 & $6-7$ & ref |NP_001166607.1 | olfactory receptor 44 [Bombyx mori] & 0.0 \\
\hline SlitOR28 & 453 & 5 & gb | ABQ84982.1 | putative chemosensory receptor 12 [Spodoptera littoralis]. & 0.0 \\
\hline SlitOR29 & 326 & 5 & ref |NP_001166894.1 | olfactory receptor 29 [Bombyx mori] & $9 e-169$ \\
\hline SlitOR30 & 239 & 4 & dbj|BAH66327.1 | olfactory receptor [Bombyx mori] & $9 e-57$ \\
\hline SlitOR31 & 400 & 5 & dbj|BAH66346.1 | olfactory receptor [Bombyx mori] & $4 e-93$ \\
\hline SlitOR32 & 205 & 4 & ref |NP_001104832.2 | olfactory receptor 16 [Bombyx mori] & 2e-101 \\
\hline SlitOR33 & 250 & 4 & ref | NP_001091785.1 | olfactory receptor 19 [Bombyx mori] & $7 e-47$ \\
\hline SlitOR34 & 141 & 2 & gb | ADM32898.1 | odorant receptor OR-5 [Manduca sexta] & $8 e-13$ \\
\hline SlitOR35 & 408 & 6 & ref | NP_001103476.1 | olfactory receptor 35 [Bombyx mori] & 1e-156 \\
\hline SlitOR36 & 245 & 4 & ref | NP_001103476.1 | olfactory receptor 35 [Bombyx mori] & $5 e-89$ \\
\hline SlitOR37 & 266 & 5 & gb|EFN70678.1| Putative odorant receptor 13a [Camponotus floridanus]. & 0.90 \\
\hline SlitGR1 & 120 & 1 & gb|EHJ69979.1| putative gustatory receptor candidate 59 [Danaus plexippus]. & $1 e-22$ \\
\hline SlitGR2 & 244 & 4 & gb|EHJ68848.1| putative Gustatory receptor 21a [Danaus plexippus]. & 3e-155 \\
\hline SlitGR3 & 213 & 4 & gb|EHJ78216.1| gustatory receptor 24 [Danaus plexippus] & $4 e-93$ \\
\hline SlitGR4 & 128 & 1 & ref | NP_001091791.1 | candidate olfactory receptor [Bombyx mori] & $2 e-26$ \\
\hline SlitGR5 & 205 & 4 & emb|CAD31947.1| putative chemosensory receptor 5 [Heliothis virescens] & $2 e-73$ \\
\hline
\end{tabular}




\section{Putative}

pheromone

receptors

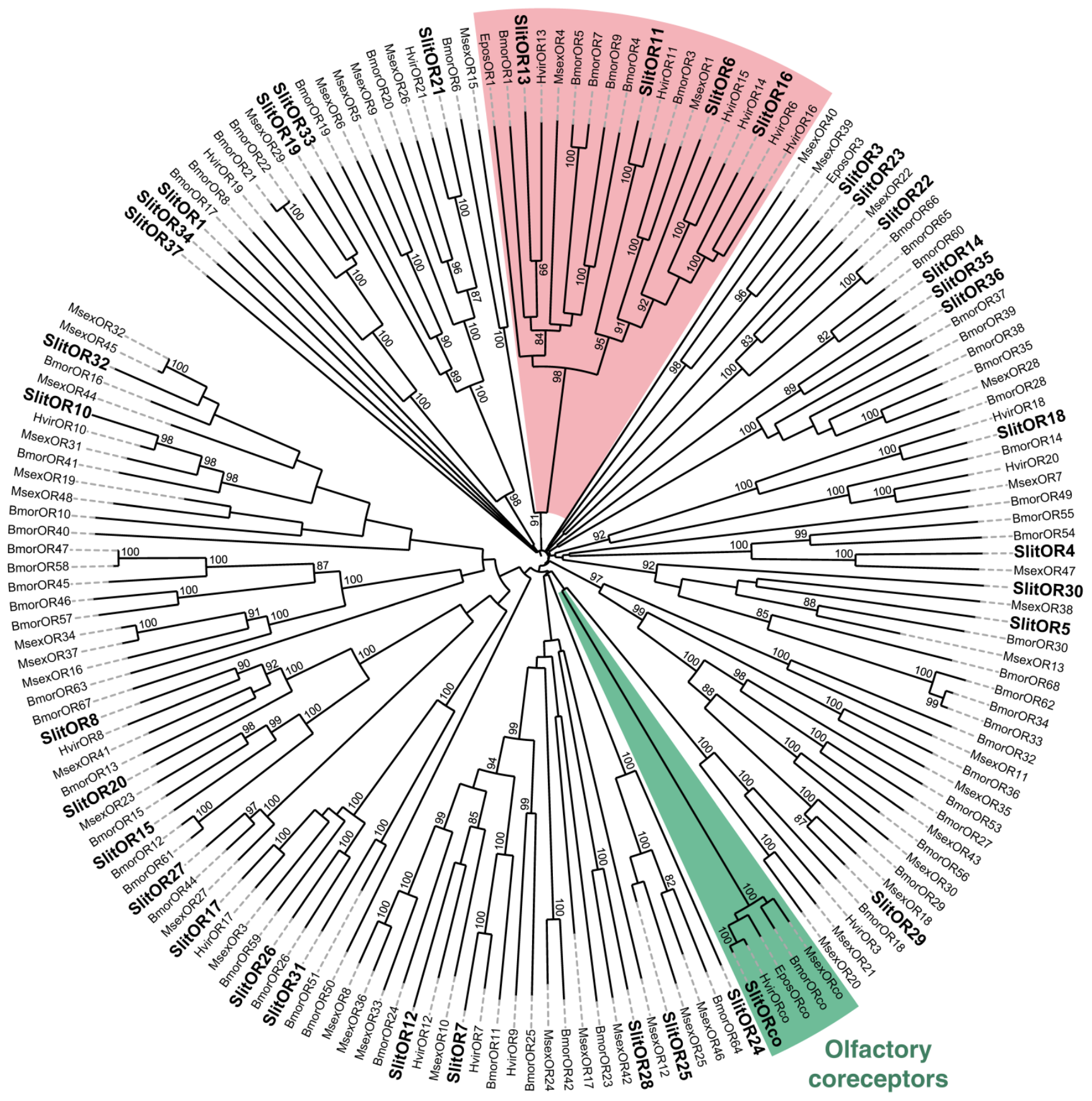

Figure 3. Neighbor-joining tree for candidate olfactory receptors (ORs) from S. littoralis and other Lepidoptera. The tree was drawn with iTOL, based on an unrooted tree constructed using the BioNJ algorithm in Seaview v.4, which was made based on a sequence alignment using ClustalW2. Bmor, B. mori [39]; Epos, E. postvittana [52], Hvir, H. virescens [40, 4I]; Msex, M. sexta [27]; Slit, S. littoralis [8](this paper). 


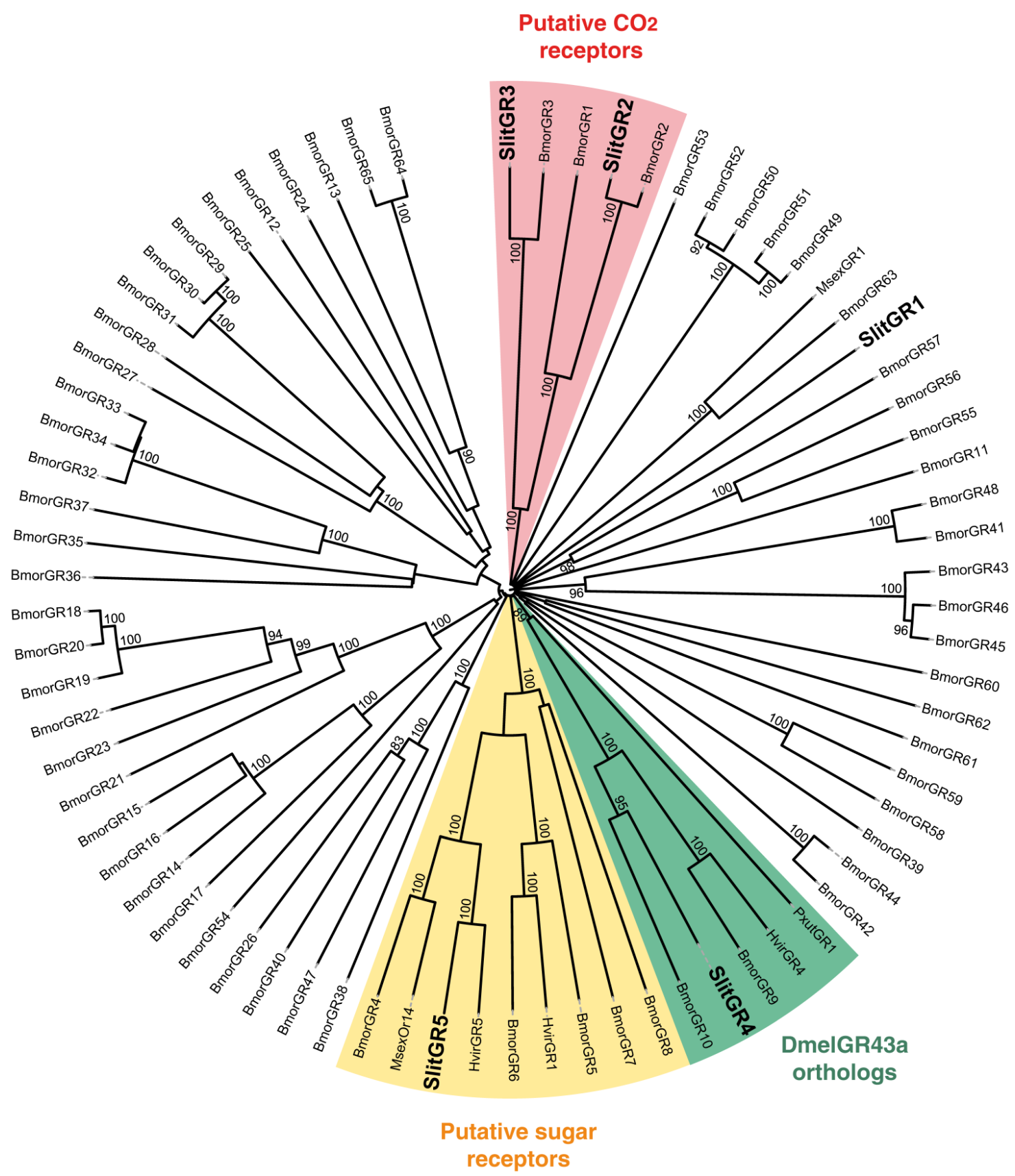

Figure 4. Neighbor-joining tree for candidate gustatory receptors (GRs) from S. littoralis and other Lepidoptera. The tree was drawn with iTOL, based on an unrooted tree constructed using the BioNJ algorithm in Seaview v.4, which was made based on a sequence alignment using ClustalW2. Bmor, B. mori [42]; Hvir, H. virescens [4I]; Msex, M. sexta [27]; Pxut, P. xuthus [43]; Slit, S. littoralis [8](this paper).

In S. littoralis, 63 glomeruli have been identified in the antennal lobe [6]. Considering the one receptor-one glomerulus paradigm [50,51], by which the number of expected ORs in a given species should correlate with the number of glomeruli in the antennal lobe, the 36 candidate OR genes identified may not represent the entire repertoire of adult $S$. littoralis ORs. Indeed, 47 and 43 ORs were identified through adult antennal transcriptome sequencing in $M$. sexta [27] and C. pomonella [28], respectively. In B. mori, 66 ORs were annotated from genome analyses [39]. However, expression studies revealed that $B$. mori adult antennae express only 35 ORs out of these 66 [39], a number that is very close to the 36 ORs we annotated in $S$. littoralis. Some glomeruli are also very likely innervated by OSNs expressing other classes of chemoreceptors, such as ionotropic receptors and gustatory receptors.

To complete our previously work [8], we conducted a preliminary study of the tissue-distribution 
of the newly identified $S$. littoralis candidate chemosensory receptors by $\mathrm{qPCR}$, and we built neighbor-joining trees with all identified SlitORs and GRs. The seven new candidate ORs were clearly antenna enriched, with antennal expression in both sexes (Fig. 5AB), supporting our annotation. In the OR phylogenetic analysis (Fig. 3), no homologs of the $B$. mori cis-jasmone receptor (BmorOR56) or the linalool/linalyl acetate receptor (BmorOR42) were found, but homologs of BmorOR29 (whose known ligands are linalool, citral and several acetates) and of the Epiphyas postvittana citral receptor (EposOR3) were present $[39,52]$. Neither were homologs of the female-enriched B. mori ORs (OR30, 45, 46, 47, 48, 50 [53, 54]) found, but SlitOR33 clustered with a $B$. mori female-specific receptor, BmorOR19, which binds linalool [53, 54]. SlitOR33 was, however, equally expressed in male and female antennae of S. littoralis [8]. None of the new sequences clustered in the pheromone receptor clade (Fig. 3).

In the GR phylogeny (Fig. 4), one of the new GR candidates (SlitGR4) grouped in the D. melanogater GR43a ortholog subgroup, which includes the newly
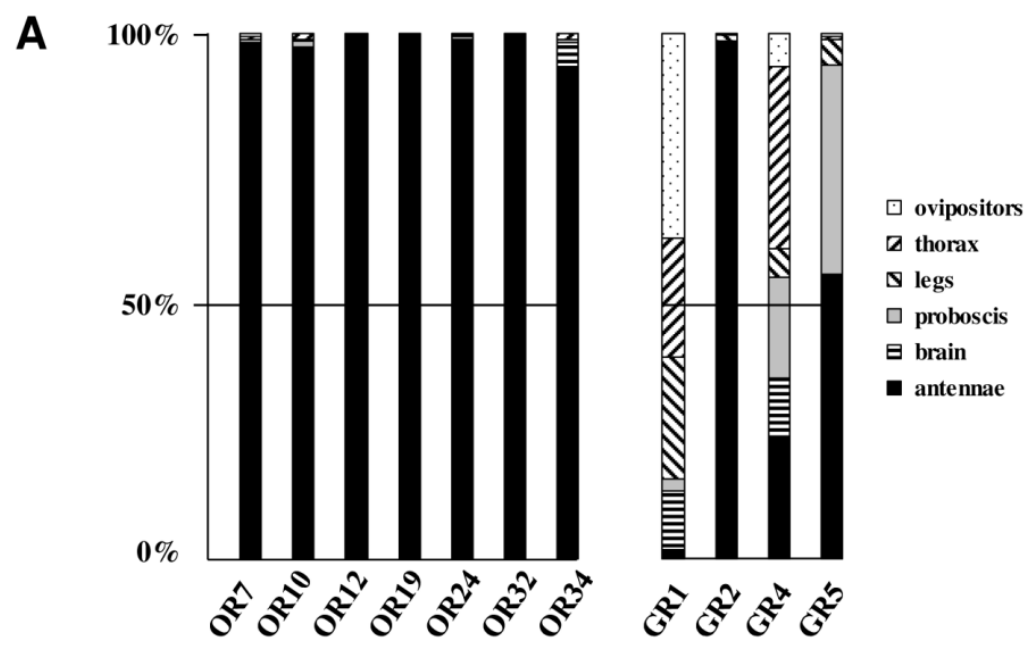

characterized B. mori fructose receptor (BmorGR9) [25] and the P. xuthus synephrine receptor (PxutGR1). The latter is expressed in female tarsi and necessary for correct oviposition behavior in this butterfly [43]. SlitGR4 appeared in our preliminary qPCR analysis as enriched in female antennae compared to male (Fig. $5 \mathrm{~B})$, and was also expressed in other female body parts such as proboscis, legs and ovipositors (Fig. 5A). Together with the fact that sugars and other carbohydrates are known to influence host preference and oviposition in female moths [55], these data suggest that SlitGR4 may fulfill an important function in $S$. littoralis female oviposition. SlitGR5 grouped in the putative sugar receptor subfamily (Fig. 4), which includes the newly characterized B. mori inositol receptor (BmorGR8) [24]. This is in concordance with previous electrophysiological studies that revealed that moth antennae are involved in sugar detection [56]. SlitGR5 was found to be expressed not only in antennae but also in female proboscis and legs (Fig. 5A). Taken together, our results suggest that sugar/oviposition site detection may involve different sensory organs. Another SlitGR (SlitGR1) did not cluster with any identified moth candidate GRs (Fig. 4). Although found in antennae, this GR was mainly expressed in ovipositors and legs (Fig. $5 \mathrm{~A})$, which the female may use to explore oviposition sites.

Figure 5. Distribution patterns of the newly identified S. littoralis candidate olfactory and gustatory receptors in different female tissues (A) and in male and female antennae (B). Gene expression levels were determined by real-time PCR and calculated relative to the expression of the rpL8 control gene and expressed as the

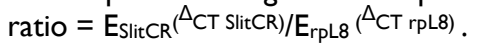

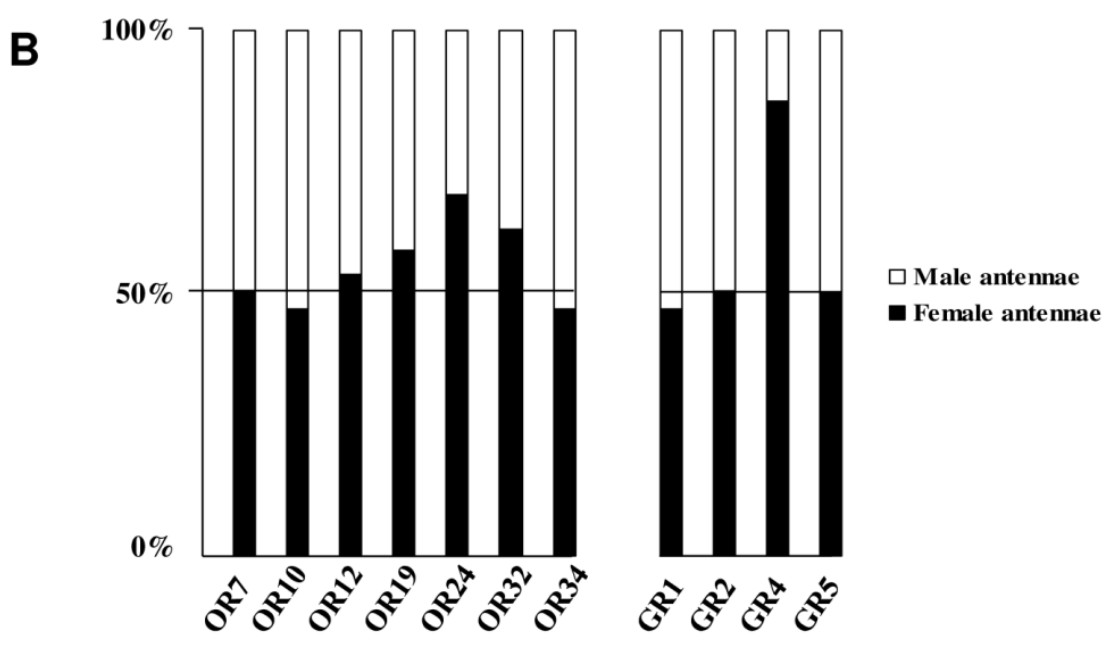


The male EST analyses allowed us to classify one candidate SlitGR (GR2, formerly named GW825869) as a putative $\mathrm{CO}_{2}$ receptor [8]. Here we could extend its sequence, confirming its high identity $(83 \%)$ with one of the three $B$. mori candidate $\mathrm{CO}_{2}$ receptors, BmGr2NJ [42]. Using qPCR, we revealed an unexpected antennal-specific expression of this gene (Fig. $5 \mathrm{~A})$. The construction of the GR tree revealed that another SlitGR, GR3, also clustered in the $\mathrm{CO}_{2}$ receptor family (Fig. 4), this one being equally expressed in antennae and proboscis [8]. Up to now, moth sensory neurons specific for $\mathrm{CO}_{2}$ have been described only on labial palps [57]. The annotation of two candidate $\mathrm{CO}_{2}$ receptors being expressed in antennae support our hypothesis that moths may also detect $\mathrm{CO}_{2}$ via their antennae.

\section{Ionotropic receptors}

We previously identified 12 candidate IRs in male antennal ESTs [58]. The sequencing of female ESTs revealed that at least seven IR genes were also expressed in female antennae (IR76b, 25a, 75q.1, 75q.2, $41 \mathrm{a}, 87 \mathrm{a}, 25 \mathrm{a})$, in concordance with our previous RT-PCR analyses [58]. No additional IR sequences could be identified in female ESTs. Thus, we yet lack the SlitIR8a sequence, a gene suspected to be expressed in insect antennae and, like IR25a, encoding a co-receptor for other IRs [23]. Both IR8a and IR25a
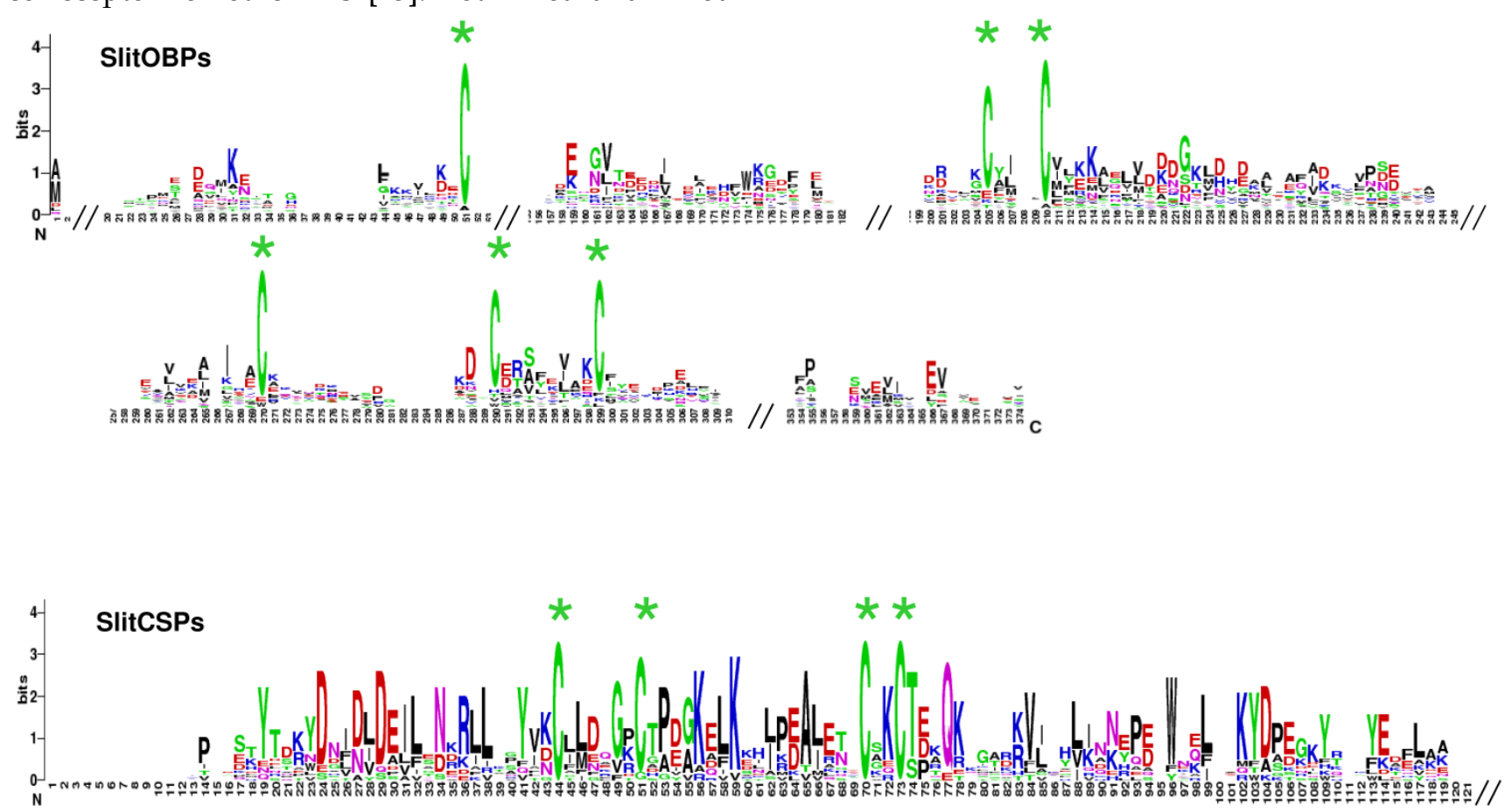

Figure 6. SlitOBP and CSP sequence logo. Degree of amino acid sequence conservation [38] along the primary sequence axis of odorant-binding proteins (OBPs) and the chemosensory proteins (CSPs) of S. littoralis. Depicted amino acid character size correlates to relative conservation across aligned sequences. Green asterisks indicate the conserved six and four cysteine motifs of OBP and CSP, respectively. could be identified in the transcriptomes of $M$. sexta [27] and C. pomonella [28].

\section{Odorant-binding proteins}

A total of 35 sequences showing similarities with Lepidoptera OBPs were identified. Comparisons with the 17 OBP dataset obtained after analysis of the male data revealed that 9 female sequences were new OBPs and one female sequence extended an existing OBP sequence. This led to a total of 26 OBPs identified in $S$. littoralis antennae (FASTA format file in Supplementary material S2). Almost all the identified genes have the characteristic hallmarks of the OBP gene family: the presence of a signal peptide, the six $\alpha$-helix pattern, and the highly conserved six cysteine profiles (Table 4, Fig. 6). However, despite the highly conserved secondary structure of OBP proteins, the SlitOBPs are highly divergent (average amino acid identity $18 \%, \min 6 \%, \max 65 \%$ ) and exhibit a wide range of protein lengths (up to 266 amino acids) and cysteine number (up to 12, Table 4). For convenience, SlitOBPs were numbered according to their closest homologs. The number of candidate SlitOBPs identified is far less than the 46 annotated OBPs found in the genome of $B$. mori $[59,60]$ but more than the 18 putative OBPs identified in the transcriptome of $M$. sexta [27]. 
Table 4. List of S. littoralis unigenes putatively involved in odorant binding. Signal peptides were determined using SignalP 4.0 [36] and $\alpha$-helices structures were predicted using the Psipred server [37].

\begin{tabular}{|c|c|c|c|c|c|c|}
\hline Name & $\begin{array}{l}\text { Length (ami- } \\
\text { no acid) }\end{array}$ & $\begin{array}{l}\text { Signal } \\
\text { peptide }\end{array}$ & $\mathrm{C}$ nb & $\begin{array}{l}\alpha \text {-helice } \\
\mathrm{nb}\end{array}$ & BlastP hit & E value \\
\hline SlitPBP1 & 164 & Yes & 6 & 7 & ABQ84981.1 pheromone-binding protein 1 [Spodoptera littoralis] & $7 e-120$ \\
\hline SlitPBP2 & 162 & Yes & 6 & 7 & AAS55551.2 pheromone binding protein 2 [Spodoptera exigua] & $2 \mathrm{e}-115$ \\
\hline SlitPBP3 & 164 & Yes & 6 & 7 & ACY78413.1 pheromone binding protein 3 [Spodoptera exigua] & $4 e-104$ \\
\hline SlitGOBP1 & 163 & Yes & 7 & 7 & ABM54823.1 general odorant-binding protein GOBP1 [Spodoptera litura] & $9 e-104$ \\
\hline SlitGOBP2 & 151 & No & 6 & 7 & ABM54824.1 general odorant-binding protein GOBP2 [Spodoptera litura] & $1 e-104$ \\
\hline SlitOBP1 & 194 & Yes & 14 & 5 & EHJ77172.1 odorant binding protein [Danaus plexippus] & $1 e-59$ \\
\hline SlitOBP2 & 184 & Yes & 8 & 6 & $\begin{array}{l}\text { CAX63249.1 odorant-binding protein SaveOBP4 precursor [Sitobion } \\
\text { avenae] }\end{array}$ & $1 e-44$ \\
\hline SlitOBP3 & 129 & Yes & 4 & 6 & ADY17884.1 odorant binding protein [Spodoptera exigua] & $8 e-72$ \\
\hline SlitOBP4 & 134 & Yes & 4 & 6 & AAL60426.1 antennal binding protein 8 [Manduca sexta] & $6 e-74$ \\
\hline SlitOBP5 & 158 & Yes & 7 & 6 & ADY17882.1 odorant binding protein [Spodoptera exigua] & $1 \mathrm{e}-108$ \\
\hline SlitOBP6 & 62 & No & 3 & 3 & NP_001140187.1 odorant-binding protein 3 precursor [Bombyx mori] & $4 e-15$ \\
\hline SlitOBP7 & 141 & Yes & 7 & $6-7$ & AEB54588.1 OBP13 [Helicoverpa armigera] & $5 e-78$ \\
\hline SlitOBP8 & 252 & Yes & 2 & 2 & BAH79159.1 odorant binding protein [Bombyx mori] & $1 e-121$ \\
\hline SlitOBP9 & 258 & Yes & 6 & 5 & ADQ01713.1 odorant binding protein 29 [Anopheles funestus] & $1 \mathrm{e}-14$ \\
\hline SlitOBP10 & 174 & No & 5 & 4 & EFA09155.1 odorant binding protein 22 [Tribolium castaneum] & $4 \mathrm{e}-10$ \\
\hline SlitOBP11 & 147 & Yes & 6 & 6 & AAR28762.1 odorant-binding protein [Spodoptera frugiperda] & $8 e-94$ \\
\hline SlitOBP12 & 147 & Yes & 6 & 6 & ADY17881.1 antennal binding protein [Spodoptera exigua] & $2 \mathrm{e}-93$ \\
\hline SlitOBP13 & 217 & Yes & 12 & 6 & AAL60414.1 twelve cysteine protein 1 [Manduca sexta] & $2 \mathrm{e}-21$ \\
\hline SlitOBP14 & 142 & Yes & 6 & 6 & AEB54586.1 OBP2 [Helicoverpa armigera] & $2 \mathrm{e}-87$ \\
\hline SlitOBP15 & 131 & No & 7 & 6 & AAL60415.1 antennal binding protein 4 [Manduca sexta] & $7 e-53$ \\
\hline SlitOBP16 & 122 & No & 9 & 5 & AAL60414.1 twelve cysteine protein 1 [Manduca sexta] & 0.040 \\
\hline SlitOBP17 & 144 & Yes & 6 & 6 & AAL60415.1 antennal binding protein 4 [Manduca sexta] & $5 e-75$ \\
\hline SlitOBP18 & 151 & Yes & 6 & 6 & AEB54589.1 OBP8 [Helicoverpa armigera] & $1 e-83$ \\
\hline SlitOBP19 & 239 & Yes & 4 & 2 & $\begin{array}{l}\text { NP_001157372.1 odorant binding protein } \mathrm{fmxg} 18 \mathrm{C} 17 \text { precursor [Bombyx } \\
\text { mori] }\end{array}$ & $1 \mathrm{e}-73$ \\
\hline SlitOBP20 & 137 & Yes & 6 & $6-7$ & CAA05508.1 antennal binding protein X [Heliothis virescens] & $5 e-75$ \\
\hline SlitOBP21 & 126 & no & 8 & 6 & AAL60413.1 antennal binding protein 3 [Manduca sexta] & $1 e-49$ \\
\hline SlitCSP1 & 128 & Yes & 4 & 6 & ACX53804.1 chemosensory protein [Heliothis virescens] & $1 \mathrm{e}-71$ \\
\hline SlitCSP2 & 120 & Yes & 4 & 6 & ACX53800.1 chemosensory protein [Heliothis virescens] & $2 \mathrm{e}-75$ \\
\hline SlitCSP3 & 75 & No & 3 & 4 & ABM67686.1 chemosensory protein CSP1 [Plutella xylostella] & $8 e-20$ \\
\hline SlitCSP4 & 148 & Yes & 5 & 5 & EHJ76401.1 chemosensory protein CSP1 [Danaus plexippus] & $2 \mathrm{e}-51$ \\
\hline SlitCSP5 & 128 & Yes & 4 & 6 & ABM67688.1 chemosensory protein CSP1 [Spodoptera exigua] & $7 e-85$ \\
\hline SlitCSP6 & 123 & Yes & 4 & 6 & ACX53806.1 chemosensory protein [Heliothis virescens] & $3 e-72$ \\
\hline SlitCSP7 & 108 & Yes & 4 & 5 & BAF91720.1 chemosensory protein [Papilio xuthus] & $1 e-56$ \\
\hline SlitCSP8 & 128 & Yes & 4 & 6 & ABM67689.1 chemosensory protein CSP2 [Spodoptera exigua] & $5 e-86$ \\
\hline SlitCSP9 & 127 & Yes & 6 & 6 & AAY26143.1 chemosensory protein CSP [Spodoptera litura] & $1 \mathrm{e}-89$ \\
\hline SlitCSP10 & 266 & Yes & 4 & $6-7$ & NP_001037069.1 chemosensory protein 9 precursor [Bombyx mori] & $2 \mathrm{e}-89$ \\
\hline SlitCSP11 & 113 & Yes & 4 & 6 & AAK14793.1 sensory appendage protein-like protein [Mamestra brassicae] & $5 e-44$ \\
\hline SlitCSP12 & 124 & Yes & 4 & 6 & ACX53817.1 chemosensory protein [Heliothis virescens] & $4 \mathrm{e}-58$ \\
\hline SlitCSP13 & 122 & Yes & 4 & 6 & ACX53813.1 chemosensory protein [Heliothis virescens] & $4 \mathrm{e}-75$ \\
\hline SlitCSP14 & 127 & Yes & 4 & 6 & BAF91712.1 chemosensory protein [Papilio xuthus] & $1 \mathrm{e}-70$ \\
\hline
\end{tabular}

\section{Sensory neuron membrane proteins and chemosensory proteins}

Sensory neuron membrane proteins (SNMPs), located in the dendritic membrane of primarily pheromone-specific OSNs, are thought to trigger ligand delivery to the receptor [61]. Moths usually express two SNMPs, and the D. melanogaster SNMP1 homolog has been demonstrated to play a role in pheromone detection [62]. The two SlitSNMPs were 
previously identified in the male ESTs, and were also represented in female ESTs, revealing that these proteins are expressed in both sexes. The SlitSNMP1 sequence was complete thanks to the male data. The female data allowed us to complete the SlitSNMP2 sequence.

Chemosensory proteins are soluble proteins expressed in a wide range of tissues and a chemosensory function has in fact been demonstrated for only a restricted number of such proteins [10], the others acting as general carriers for hydrophobic ligands in the insect body. Here, we identified 17 sequences expressed in female antennae showing similarities with Lepidoptera CSPs. Comparison with the 9 CSP annotated in the male ESTs revealed that five female sequences were new CSPs, leading to a total of 14 CSPs identified in S. littoralis antennae (FASTA format file in Supplementary material S2). Almost all deduced protein sequences have the characteristic hallmarks of CSPs: the presence of a signal peptide, the six $\alpha$-helix pattern, and the highly conserved four cysteine profiles (Table 4, Fig. 6). 22 putative CSPs have been annotated in B. mori $[60,63]$ and 21 in M. sexta [27], while we identified only 14 candidate CSPs. Nevertheless, our data confirmed that Lepidoptera express a higher number of CSPs than other insect families, such as Diptera [60]. Although their role in chemoreception should be taken with caution, at least one SlitCSP (SlitCSP3) may be involved in contact chemoreception since it is homologous to Plutella xylostella CSP1 that binds non volatile oviposition deterrents [64].

\section{LepidoDB implementation}

Lepido-DB (http://www.inra.fr/lepidodb) is a centralized bioinformatic resource for the genomics of major lepidopteran pests [65]. This Information System was designed to store, organize, display and distribute various genomic data and annotations. All the data, unigenes, ORFs and their annotation generated in this project have been included in LepidoDB. As a result, from the project page http://www.inra.fr/lepidodb/spodoptera_littoralis, one can retrieve the whole sequence set, query with a keyword and retrieve the corresponding sequences.

\section{Conclusion}

The main objective of this study was to investigate the antennal transcriptome of the noctuid moth $S$. littoralis. Thanks to the sequencing of female ESTs, we annotated a total of 26 candidate OBPs, 14 CSPs, 36 ORs and 5 GRs in the antennae of S. littoralis. This strategy appears to be particularly relevant for the identification of new insect chemosensory receptors in a species for which no genomic data are available. The availability of this large antennal transcriptome constitutes a valuable resource for studies of insect olfaction.

Although the generation of gender-specific transcriptomes did not highlight strong differences between sexes, we evidenced male-specific ORs as candidate pheromone receptors and a female-enriched GR as a candidate oviposition stimulant receptor. It has to be noticed that we focused our study on naïve insects, whereas mating is known to induce big changes in the olfactory behaviors. For example, S. littoralis females switch their olfactory response from food to egg-laying cues following mating [66]. This switch may be associated with regulation in the transcriptome expression. Comparison of antennal transcriptomes from males and females that encountered diverse experiences would then lead to identification of more regulated genes, as candidate genes involved in gender-specific behaviors.

\section{Supplementary Material}

S1: Comparison of male and female gene expression using GO categorization for biological processes (upper) and molecular function (lower), both level 3. S2: Fasta amino acid sequences of annotated S. littoralis OBPs, CSPs, ORs and GRs. http://www.biolsci.org/v08p1036s1.pdf

\section{Acknowledgements}

We thank the BioGenouest platform for their bioinformatics support. This work was supported by INRA, ANR-09-BLAN-0239-01 funding, a Genoscope partnership, the Swedish Government Linnaeus initiative Insect Chemical Ecology, Ethology and Evolution, the Swedish Science Council, the Trygger Foundation, and the Max Planck Society.

\section{Competing Interests}

The authors have declared that no competing interest exists.

\section{References}

1. Kaissling K-E. Physiology of pheromone reception in insects (an example of moths). ANIR. 2004; 6: 73-91.

2. Nesbitt BF, Beevor PS, Cole RA, Lester R, Poppi RG. Sex pheromones of two noctuid moths. Nat New Biol. 1973; 244: 208-9.

3. Anderson P, Hansson BS, Lofqvist J. Plant-odour-specific receptor neurones on the antennae of female and male Spodoptera littoralis. Physiol Entomol. 1995; 20: 189-98.

4. Ljungberg $\mathrm{H}$, Anderson P, Hansson BS. Physiology and morphology of pheromone-specific sensilla on the antennae of male and female Spodoptera littoralis (Lepidoptera: Noctuidae). J Insect Physiol. 1993; 39: 253-60.

5. Ochieng SA, Anderson P, Hansson BS. Antennal lobe projection patterns of olfactory receptor neurons involved in sex pheromone detection in Spodoptera littoralis (Lepidoptera: Noctuidae). Tissue Cell. 1995; 27: 221-32.

6. Couton L, Minoli S, Kieu K, Anton S, Rospars JP. Constancy and variability of identified glomeruli in antennal lobes: computational approach in Spodoptera littoralis. Cell Tissue Res. 2009; 337: 491-511. 
7. Sjoholm M, Sinakevitch I, Strausfeld NJ, Ignell R, Hansson BS. Functional division of intrinsic neurons in the mushroom bodies of male Spodoptera littoralis revealed by antibodies against aspartate, taurine, FMRF-amide, Mas-allatotropin and DC0. Arthropod Struct Dev. 2006; 35: 153-68.

8. Legeai F, Malpel S, Montagne N, Monsempes C, Cousserans F, Merlin C, et al. An Expressed Sequence Tag collection from the male antennae of the Noctuid moth Spodoptera littoralis: a resource for olfactory and pheromone detection research. BMC Genomics. 2011; 12: 86.

9. Vogt RG. Biochemical diversity of odor detection:OBPs, ODEs and SNMPs. In: Blomquist GJ, Vogt RG, editors. Insect Pheromone Biochemistry and Molecular Biology. Elsevier Academic Press. 2003: 391-445.

10. Pelosi P, Zhou JJ, Ban LP, Calvello M. Soluble proteins in insect chemical communication. Cell Mol Life Sci. 2006; 63: 1658-76.

11. Jacquin-Joly E, Vogt RG, Francois MC, Nagnan-Le Meillour P. Functional and expression pattern analysis of chemosensory proteins expressed in antennae and pheromonal gland of Mamestra brassicae. Chem Senses. 2001; 26: 833-44.

12. Briand L, Swasdipan N, Nespoulous C, Bezirard V, Blon F, Huet JC, et al. Characterization of a chemosensory protein (ASP3c) from honeybee (Apis mellifera L.) as a brood pheromone carrier. Eur J Biochem. 2002; 269: 4586-96.

13. Kitabayashi AN, Arai T, Kubo T, Natori S. Molecular cloning of cDNA for p10, a novel protein that increases in the regenerating legs of Periplaneta americana (American cockroach). Insect Biochem Mol Biol. 1998; 28: 785-90.

14. Gonga L, Luoa Q, Rizwan-ul-Haqa M, Hua M-Y. Cloning and characterization of three chemosensory proteins from Spodoptera exigua and effects of gene silencing on female survival and reproduction. Bull Entomol Res. 2012; doi:10.1017/S0007485312000168.

15. Touhara K, Vosshall LB. Sensing odorants and pheromones with chemosensory receptors. Annu Rev Physiol. 2009; 71: 307-32.

16. Benton R, Vannice KS, Gomez-Diaz C, Vosshall LB. Variant ionotropic glutamate receptors as chemosensory receptors in Drosophila. Cell. 2009; 136: 149-62.

17. Larsson MC, Domingos AI, Jones WD, Chiappe ME, Amrein H, Vosshall LB. Or83b encodes a broadly expressed odorant receptor essential for Drosophila olfaction. Neuron. 2004; 43: 703-14.

18. Benton R, Sachse S, Michnick SW, Vosshall LB. Atypical membrane topology and heteromeric function of Drosophila odorant receptors in vivo. PLoS Biol. 2006; 4: e20.

19. Vosshall LB, Hansson BS. A Unified Nomenclature System for the Insect Olfactory Coreceptor. Chem Senses. 2011; 36: 497-8.

20. Wicher D, Schafer R, Bauernfeind R, Stensmyr MC, Heller R, Heinemann $\mathrm{SH}$, et al. Drosophila odorant receptors are both ligand-gated and cyclic-nucleotide-activated cation channels. Nature. 2008; 452: 1007-11.

21. Sato K, Pellegrino M, Nakagawa T, Vosshall LB, Touhara K. Insect olfactory receptors are heteromeric ligand-gated ion channels. Nature. 2008; 452: 1002-6

22. Croset V, Rytz R, Cummins SF, Budd A, Brawand D, Kaessmann H, et al. Ancient protostome origin of chemosensory ionotropic glutamate receptors and the evolution of insect taste and olfaction. PLoS Genet. 2010;6(8):e1001064

23. Abuin L, Bargeton B, Ulbrich MH, Isacoff EY, Kellenberger S, Benton R. Functional architecture of olfactory ionotropic glutamate receptors. Neuron. 2011; 69: 44-60.

24. Zhang HJ, Anderson AR, Trowell SC, Luo AR, Xiang ZH, Xia QY. Topological and functional characterization of an insect gustatory receptor. PLoS ONE. 2011; 6: e24111.

25. Sato K, Tanaka K, Touhara K. Sugar-regulated cation channel formed by an insect gustatory receptor. Proc Natl Acad Sci U S A. 2011; 108: 11680-5.

26. Jordan MD, Stanley D, Marshall SD, De Silva D, Crowhurst RN, Gleave $\mathrm{AP}$, et al. Expressed sequence tags and proteomics of antennae from the tortricid moth, Epiphyas postvittana. Insect Mol Biol. 2008; 17: 361-73.

27. Grosse-Wilde E, Kuebler LS, Bucks S, Vogel H, Wicher D, Hansson BS. Antennal transcriptome of Manduca sexta. Proc Natl Acad Sci U S A. 2011; 108: 7449-54.

28. Bengtsson JM, Trona F, Montagné N, Anfora G, Ignell R, Witzgall P, et al. Putative chemosensory receptors of the codling moth, Cydia pomonella, identified by antennal transcriptome analysis. PLoS ONE. 2012; $7(2): \mathrm{e} 31620$.

29. Poitout $S$, Buès R. Elevage de chenilles de vingt-huit espèces de Lépidoptères Noctuidae et de deux espèces d'Arctiidae sur milieu artificiel simple. Particularités de l'élevage selon les espèces. Ann Zool Ecol anim. 1974; 6: 431-41.
30. Gouzy J, Carrere S, Schiex T. FrameDP: sensitive peptide detection on noisy matured sequences. Bioinformatics. 2009; 25: 670-1.

31. Conesa A, Gotz S, Garcia-Gomez JM, Terol J, Talon M, Robles M. Blast2GO: a universal tool for annotation, visualization and analysis in functional genomics research. Bioinformatics. 2005; 21: 3674-6.

32. Quevillon E, Silventoinen V, Pillai S, Harte N, Mulder N, Apweiler R, et al. InterProScan: protein domains identifier. Nucleic Acids Res. 2005; 33: W116-20.

33. Bluthgen N, Brand K, Cajavec B, Swat M, Herzel H, Beule D. Biological profiling of gene groups utilizing Gene Ontology. Genome Inform. 2005; 16: 106-15.

34. Altschul SF, Madden TL, Schaffer AA, Zhang J, Zhang Z, Miller W, et al. Gapped BLAST and PSI-BLAST: a new generation of protein database search programs. Nucleic Acids Res. 1997; 25: 3389-402.

35. Krogh A, Larsson B, von Heijne G, Sonnhammer EL. Predicting transmembrane protein topology with a hidden Markov model: application to complete genomes. J Mol Biol. 2001; 305: 567-80.

36. Petersen TN, Brunak S, von Heijne G, Nielsen H. SignalP 4.0: discriminating signal peptides from transmembrane regions. Nat Methods. 2011; 8: 785-6.

37. Buchan DW, Ward SM, Lobley AE, Nugent TC, Bryson K, Jones DT. Protein annotation and modelling servers at University College London. Nucleic Acids Res. 2010; 38: W563-8.

38. Crooks GE, Hon G, Chandonia JM, Brenner SE. WebLogo: a sequence logo generator. Genome Res. 2004; 14: 1188-90.

39. Tanaka K, Uda Y, Ono Y, Nakagawa T, Suwa M, Yamaoka R, et al. Highly selective tuning of a silkworm olfactory receptor to a key mulberry leaf volatile. Curr Biol. 2009; 19: 881-90.

40. Krieger J, Grosse-Wilde E, Gohl T, Dewer YM, Raming K, Breer H. Genes encoding candidate pheromone receptors in a moth (Heliothis virescens). Proc Natl Acad Sci USA. 2004; 101: 11845-50.

41. Krieger J, Raming K, Dewer YM, Bette S, Conzelmann S, Breer H. A divergent gene family encoding candidate olfactory receptors of the moth Heliothis virescens. Eur J Neurosci. 2002; 16: 619-28.

42. Wanner KW, Robertson HM. The gustatory receptor family in the silkworm moth Bombyx mori is characterized by a large expansion of a single lineage of putative bitter receptors. Insect Mol Biol. 2008; 17: 621-9.

43. Ozaki K, Ryuda M, Yamada A, Utoguchi A, Ishimoto H, Calas D, et al. A gustatory receptor involved in host plant recognition for oviposition of a swallowtail butterfly. Nat Commun. 2011; 2: 542.

44. Larkin MA, Blackshields G, Brown NP, Chenna R, McGettigan PA, McWilliam $H$, et al. Clustal $W$ and Clustal $X$ version 2.0. Bioinformatics. 2007; 23: 2947-8.

45. Gouy M, Guindon S, Gascuel O. SeaView version 4: A multiplatform graphical user interface for sequence alignment and phylogenetic tree building. Mol Biol Evol. 2010; 27: 221-4.

46. Letunic I, Bork P. Interactive Tree Of Life (iTOL): an online tool for phylogenetic tree display and annotation. Bioinformatics. 2007; 23: 127-8.

47. Pfaffl MW. A new mathematical model for relative quantification in real-time RT-PCR. Nucleic Acids Res. 2001; 29: e45.

48. Jacquin-Joly E, Merlin C. Insect olfactory receptors: contributions of molecular biology to chemical ecology. J Chem Ecol. 2004; 30: 2359-97.

49. ISGC. The genome of a lepidopteran model insect, the silkworm Bombyx mori. Insect Biochem Mol Biol. 2008; 38: 1036-45.

50. Couto A, Alenius M, Dickson BJ. Molecular, anatomical, and functional organization of the Drosophila olfactory system. Curr Biol. 2005; 15: 1535-47.

51. Fishilevich E, Vosshall LB. Genetic and functional subdivision of the Drosophila antennal lobe. Curr Biol. 2005; 15: 1548-53.

52. Jordan MD, Anderson A, Begum D, Carraher C, Authier A, Marshall SD, et al. Odorant receptors from the light brown apple moth (Epiphyas postvittana) recognize important volatile compounds produced by plants. Chem Senses. 2009; 34: 383-94.

53. Wanner KW, Anderson AR, Trowell SC, Theilmann DA, Robertson HM, Newcomb RD. Female-biased expression of odourant receptor genes in the adult antennae of the silkworm, Bombyx mori. Insect Mol Biol. 2007; 16: 107-19.

54. Anderson AR, Wanner KW, Trowell SC, Warr CG, Jaquin-Joly E, Zagatti $\mathrm{P}$, et al. Molecular basis of female-specific odorant responses in Bombyx mori. Insect Biochem Mol Biol. 2009; 39: 189-97.

55. Lombarkia N, Derridj S. Resistance of apple trees to Cydia pomonella egg laying due to leaf surface metabolites. Ent Exp Appl. 2008; 128: 57-65.

56. Jorgensen K, Almaas TJ, Marion-Poll F, Mustaparta H. Electrophysiological characterization of responses from gustatory receptor neurons of sensilla chaetica in the moth Heliothis virescens. Chem Senses. 2007; 32: 863-79. 
57. Bogner F, Boppre M, Ernst KD, Boeckh J. CO2 sensitive receptors on labial palps of Rhodogastria moths (Lepidoptera: Arctiidae): physiology, fine structure and central projection. J Comp Physiol A. 1986; 158: 741-9.

58. Olivier V, Monsempes C, Francois MC, Poivet E, Jacquin-Joly E. Candidate chemosensory ionotropic receptors in a Lepidoptera. Insect $\mathrm{Mol} \mathrm{Bi-}$ ol. 2011; 20: 189-99.

59. Gong DP, Zhang HJ, Zhao P, Xia QY, Xiang ZH. The Odorant Binding Protein gene family from the genome of silkworm, Bombyx mori. BMC Genomics. 2009; 10: 332.

60. Vieira FG, Rozas J. Comparative genomics of the odorant-binding and chemosensory protein gene families across the Arthropoda: origin and evolutionary history of the chemosensory system. Genome Biol Evol. 2011; 3: 476-90.

61. Nichols Z, Vogt RG. The SNMP/CD36 gene family in Diptera, Hymenoptera and Coleoptera: Drosophila melanogaster, D. pseudoobscura, Anopheles gambiae, Aedes aegypti, Apis mellifera, and Tribolium castaneum. Insect Biochem Mol Biol. 2008; 38: 398-415.

62. Benton R, Vannice KS, Vosshall LB. An essential role for a CD36-related receptor in pheromone detection in Drosophila. Nature. 2007; 450: 289-93.

63. Gong DP, Zhang HJ, Zhao P, Lin Y, Xia QY, Xiang ZH. Identification and expression pattern of the chemosensory protein gene family in the silkworm, Bombyx mori. Insect Biochem Mol Biol. 2007; 37: 266-77.

64. Liu X, Luo Q, Zhong G, Rizwan-Ul-Haq M, Hu M. Molecular characterization and expression pattern of four chemosensory proteins from diamondback moth, Plutella xylostella (Lepidoptera: Plutellidae). J Biochem 2010; 148: 189-200.

65. d'Alençon E, Sezutsu H, Legeai F, Permal E, Bernard-Samain S, Gimenez $\mathrm{S}$, et al. Extensive synteny conservation of holocentric chromosomes in Lepidoptera despite high rates of local genome rearrangements. Proc Natl Acad Sci U S A. 2010; 107: 7680-5.

66. Saveer AM, Kromann SH, Birgersson G, Bengtsson M, Lindblom T, Balkenius A, et al. Floral to green: mating switches moth olfactory coding and preference. Proc Biol Sci. 2012;279: 2314-22. 